reason to expect $a$ greater risoconstriction at the bleeding point than elsewhere and a rise in the general blood pressure beyond that necessary to sustain the circulation in the medulla, is contraindicated in hemorrhage. This, of course, does not apply to postpartum hemorrhage, because ergot has a specific action on the uterus, causing a firm contraction and thereby closing the bleeding vessels by compression.

Sollmann and Brown have recently studied the action of ergot when injected intravenously into dogs. 'They found that it influenced the general circulation but little, particularly when the blood pressure was much lowered. They found that it caused a temporary fall in the blood pressure with speedy recovery, generally followed by an unimportant rise. The effects were mainly due to the action on the heart, as the vasomotor action was very slight.

The further discussion of its obstetrical use does not belong here.

Erigota.-U. S.--The sclerotium of Claviceps purpurca, gathered from rye, and not more than one year old.

While ersot had been used in Germany for centuries as a household remedy, it was not generally recognized as an efficient drug until some time after 1777, when Desgranges published several essays on the use of ergot.

The vasoconstrictor properties of this drug appear to be due to the alkaloid cornutin, discovered by Kobert in 1884, and more fully described by Keller in 1894 .

Extractum Ergote:-U. S.-This is a hydro-alcoholic extract of ergot partially purified by the addition of hydrochloric acid subsequently neutralized with sodium carbonate. It should represert eight times its weight of ergot.

Average dose: $0.25 \mathrm{gm}$. (4 grains).

Fluidextractum Frgotas.-U. S.-This is directed to be made with diluted alcohol that has been acidified by the addition of acetic acid.

Average dose: 2 c.c. ( 30 minims).

VInUM Ergote.-This represents 20 per cent. of the fluidextract of ergot in fortified white wine.

Average dose: 8 c.c. ( 2 fluidrams).

Ergot is preferably given in the form of the fluid extract without other additions. There are a number of water-soluble preparations on the market at the present time that are intended for hypodermic use, which, it is claimed, possess all the active principles of ergot, without the odorous and nauseating contaminations.

\section{New Instruments}

\section{SOME MASTOID INSTRUMENTS. AND SOME APPLIANCES FOR EAR WORK. \\ FRANK ALLPORT, M.D. CHICAgo. \\ A MidDLE EAR SYRINGE.}

Practically all middle ear syringes are unclean and frequently out of order. I submit one of glass ana rubber that can always be ready for use, and which is so cheap that the surgeon can afford to possess a number of them (Fig. 1). An objection has been raised to it that the point is not large enough to enter minute perforations of the drumhead. In answer to this I contend that no middle ear should be irrigated through a minute drumhead perforation. Such tympanic irrigations should be confined to those cases where the irrigating liquid can find a ready exit, otherwise dangerous complications are likely to ensue.

\section{A MASTOID PERIOSTEOTOME.}

In stripping the mastoid process of its periosteum after the primary incision has been accomplished, an effort should be made to preserve all possible periosteum. This purpose is liable to be defeated by the sharp scraping instruments usually employed, which tear and mangle the periosteum. I desire to recommend the instrument shown in Figure 2 as one that can be passed underneath the periosteum, thus lifting and not tearing it from the bone. It has a small end and a large

4. The Jovryal A. M. A., July 22, 1905, p. 229. end, and the latter should be used where much force is indicated. This instrument can not be used when the lower portion of the mastoid is to be cleaned, for at this portion of the bone the roughened surface and tendinous attachments of the muscles make the soft tissue cling to the irregular bone surface with such tenacity that one of the scraping periosteotomes and a pair of strong scissors must be used. My instrument, however, can be used at all other portions of the bone.

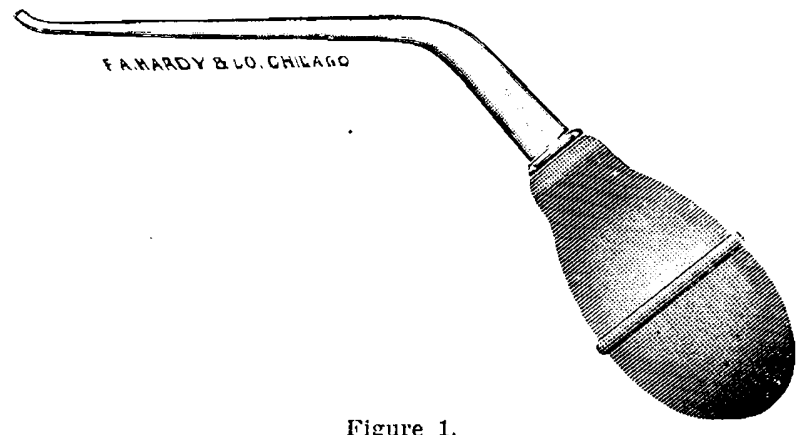

The narrow end of the instrument has been gently concavoconvexed to fit the bony external auditory canal, and is used in the radical mastoid operation to pass between the cartilaginous and the bony meati, in order to separate them thoroughly and facilitate the subsequent steps of the operation. This instrument is not useful in secondary mastoid operation. where dense cicatricial bands connect the soft tissue with the bone.

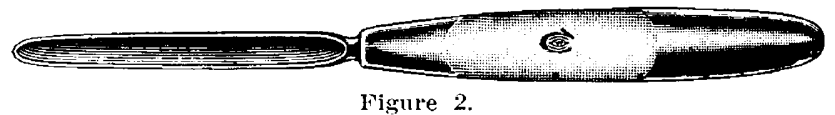

A PERMANENT NEEDLE.

Having been much troubled with the breaking of neetles in closing mastoid wounds, I have for a long time used the instrument shown in the drawing (Fig. 3). Near the point of the needle is a small opening, that can be opened and closed by pressing the button near the handle. The needle is pushed through the soft tissue with the small perforation closed, the suture is then doubled and placed in the opening, which is

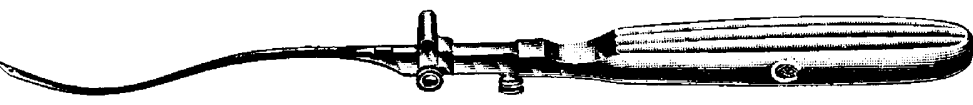

Figure 3 .

then elosed. The needle is then withdrawn with the suture and one end of the suture pulled through when the suture will be ready for tying. 1 use this instrument only in $\mathrm{my}$ buried catgut sutures in the radical operation, as for closing the outside lips of the wound I use the metal sutures proposed by Michel.

THE MTCHEL SUTURES, ETC.

For elosing the external lips of the mastoid wound I have

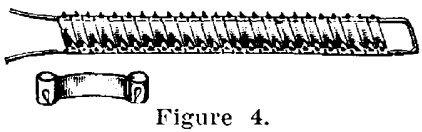

for some time been lising with great satisfaction the metal Michel suture, a drawing of which is here given (Fig. 4).

These sutures are easily and quickly applied, scarcely ever produce stitch abscesses, and result in a quicker healing and a more imperceptible line of cicatrix than any sutures $I$ have ever employed. Several instruments or appliances are neces-

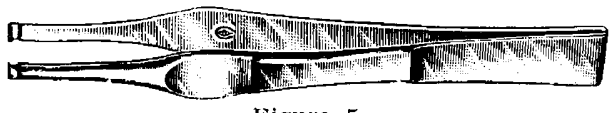

Figure 5.

sary for their advantageous employment, riz., a wire suture holder which enables the surgeon to conveniently take hold of the suture, a strong pair of forceps (Fig. 5) with points so constructed as to grasp and hold firmly the suture and to 
grasp and hold the two sides of the wound forcefully, and a pair of forceps (Fig. 6) for removing the sutures after the wound has healed. The two ends of the removing forceps are pointed so that one end may be gently insinuated underneath the middle of the bent suture after it has been holding the soft tissues together for a few days. The two blades of the forceps are then brought firmly together at the middle of the suture, one blade being underneath and the other over the suture; this, of course, will cause the two outer ends of the suture to separate, thus removing the sharp points from the

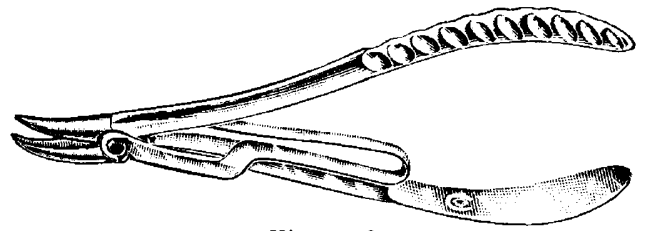

Figure 6.

flesh. The suture is now liberated and may be cast aside or straightened out and used again.

In applying a suture it should be held on the wire loop or suture holder by an assistant, the surgeon should grasp the suture with the forceps and pull it off from the loop, an assistant should grasp the two lips of the wound with strong forceps, bring them together and hold them up, thus approximated, as prominently as possible for the benefit and convenience of the surgeon, who should now forcefully clamp the suture around the lips of the wound, and bring the two

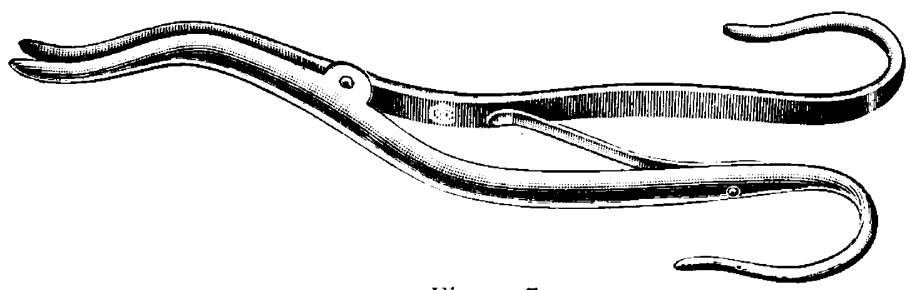

Figure 7 .

ends of the suture, armed with the little needle points, as close together as possible by firm pressure of the strong for. ceps. Five or six sutures are usually sufficient, even in a large mastoid wound. I usually remove about half the sutures in three days and the remainder in six or even days.

\section{a meatal divulisor.}

For those who split the cartilaginous meatus after the method of Panse in producing healing flaps after the radical mastoid operation the instrument here shown will prove of value (Fig. 7). A grooved director is used by some to guide

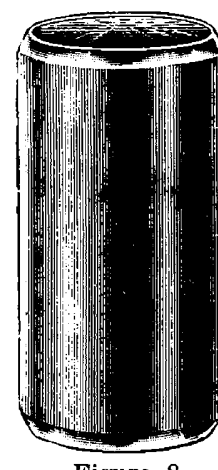

Figure 8.

the knife, strong forceps, widely spread, by others, but for a long time I have used the "meatal divulsor" as I call it. The instrument is introduced through the meatus and passed through to its further end and spread so that the cartilaginous meatus is placed on a stretch. A knife can now be passed into the tympanic end of the meatus and the cartilaginous wall easily and accurately severed up to the desired point at the conchal rim, spreading the divulsor more and more as the incision advances toward the concha.
A Metal Covered WOOden MaLlet.

For mastoid work a silver or lead mallet is too heavy and harsh, and a rawhide mallet is too soft and soon becomes useless from boiling; a wood mallet also becomes cracked and worthless from boiling. I employ, therefore, a good-sized mallet made of lignum vitæ, encased in steel, with a strong steel handle. It is imperishable and thoroughly satisfactory (Fig. 8).

\section{A BONE CRESHER.}

An important step in the radical mastoid operatio is the breaking down of the posterior wall of the meatus. Injury to the facial rerve, semicircular canal, ete., may follow this procedure. The complete clearing away of the upper portion of the bony meatus, without injury to surrounding parts, is much facilitated if a small pathway through the bone to the tympanic cavity, etc., can be distinctly blazed by some instrument. I have found the "bone crusher" (Fig. 9), to be of

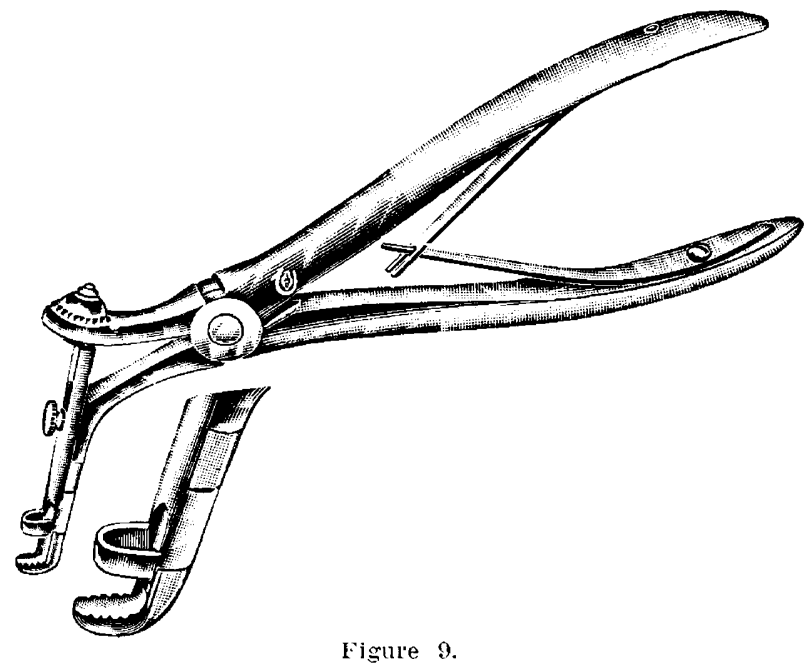

great service for this purpose. Before using it the mastoid cavity, antrum, ete., should be thoroughly curetted and cleaned. The crusher has not sufficient power and strength (and should not have) to break down the entire thickness of meatal wall. The surgeon should proceed, therefore, as if expecting to destroy the meatal wall with the chisel and mallet alone, and carve away a considerable portion of the bony wall with these instruments. After, perhaps, one-half of the work has been accomplished, the footplate of the crusher should be carefully insinuated through the antrum to the middle ear, the handle firmly depressed so as to raise the foot. plate of the instrument away from the inner tympanic wall, and the arms of the handle forcefully brought together, which will, of course, crush down the bone contained between the jaws of the instrument. The blades are not broad, but narrow, and the channel thus produced is, therefore, of small dimensions, but it is sufficient to point the way for further bone destruction, which can now be easily and safely accomplished by chisel, rongeur and curette.

\section{TWO KNIVES.}

One of the recognized defects in the radical mastoid operation has been the deformity often produced by an abnormally wide and gaping meatus. This trouble I have been able to dissipate entirely by the use of two knives, which I will now describe, my description referring, of course, as will be seen, to the Panse flap which $I$ use to the exclusion of all others. After everything is ready for flap making and wound closure I insert the double-edged knife (Fig. 10) into the raw posterior portion of the auricle, as near as possible to the middle edge of the meatus. The knife is carefully pushed forward 
and made to reappear at the middle of the meato-conchal edge; after enlarging the opening a little the knife is withdrawn and the blunt-pointed knife (Fig. 11) is made to take its place, as the seesaw movement necessary to finish the incision up and down would be likely to produce multiple punctures on the other side of the meatus. The blunt point of the

\section{Figure 10.}

other knife renders the subsequent procedures free from such embarrassments, and the fact that the blade convexes forward on the edge enables the surgeon to complete more effectively the remote corners of the incision. The knife should be carried up as far as desired, anc then down, always keeping in view the meato-conchal edge, and keeping the line of incision exactly on this edge. In this way the meatal opening is enlarged only a trifle, and the surgeon can be absolutely sure

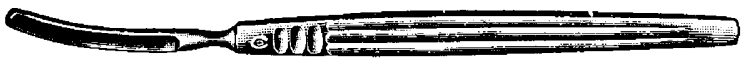

Figure 11.

that a constant and non-deforming result will occur. The backward incision along the cartilaginous meatus can now be completed by the divulsor and knife as already described.

\section{A GAUZE PACKER.}

A good instrument for packing gauze is desirable not only at the time of the mastoid operation, but more particularly at the subsequent dressings. I have already described the packer here shown, but as two important modifications have been made in it, $I$ venture to refer to it again. It has a coneshaped point, flat and round at the distal extremity, which

\section{(1)}

Figure 12.

gradually diminishes in bulk as the shank of the instrument is reached, thus enabling the operator to pack down firmly the gauze without any tendency to displace the packing as the instrument is withdrawn. The narrow portion of the shank as formerly made was flexible; it is now stiff and firm. The distal end used to be smooth; it is now roughened and catches the gauze better.

\section{A HOOK PROBE.}

I have described this hook probe some years ago as a useful instrument for finding and dislocating the ossicles in the

Figure 13

operation for ossiculectomy, and also as a generally useful ear hook and probe. I now, however, find it exceedingly efficacious in the mastoid operation as a probe for the detection of carious spots, and especially for locating openings in the roof of the tympanum, aditus, or antrum. It consists of a slender shank, bent at right angles at the end and tipped with a small round ball (Fig. 13).

TIIE MASTOID RETRACTOR.

I trust I may be pardoned for referring again to my mastoid retractors, now in general use for so many years (Fig. 14). They have been modified somewhat of late by making them much stronger so that they possess great power and do not break. The skin incision should be long enough to allow the operator to place two pairs of these retractors in the opening, one pair in the upper angle of the wound and another pair in the lower angle. The teeth of the arms of the retractors

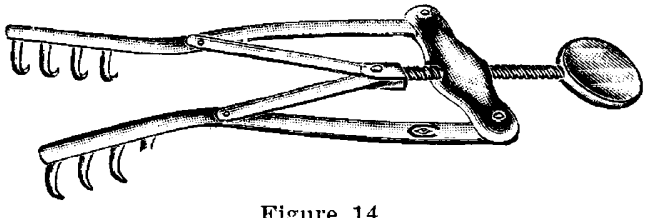

Figure 14.

should be placed underneath the periosteum and the arms should then be expanded to their utmost limits by turning the thumbscrew at the base of the instrument. I rarely use an artery forceps, and when both retractors are thus widely separated a perfect and comparatively bloodless field of operation is observed between the four arms of the two retractors. Some operators still employ the old hand retractors, one for each side of the wound, but these retractors necessitate extra hands and arms around the operative field, and they do not control hemorrhage, and no one can always maintain steady and uniform hand retraction.

\section{a pillar separator.}

Robertson's pillar separator usually acts well in separating the pillar from the tonsil preparatory to a removal of the lat-

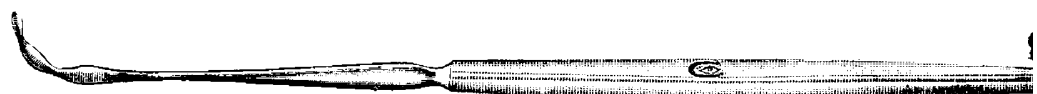

Figure 16.

ter, but it does not always cut well, which is embarrassing to the operator and frequently painful to the patient on account of the forcible dragging of the blade on the yielding soft tissue. I beg to suggest a knife with a wavy blade, like a bread knife, which, when sharp, by the use of a gentle to-andfro movement, easily and comparatively painlessly cuts the soft tissue without much effort (Fig. 16).

\section{A BEDSIDE TRAY.}

It would seem easy to purchase a small and convenient tray for carrying a few eye and ear instruments, solutions, cotton, etc., from bed to bed in a hospital, and yet a faithful search

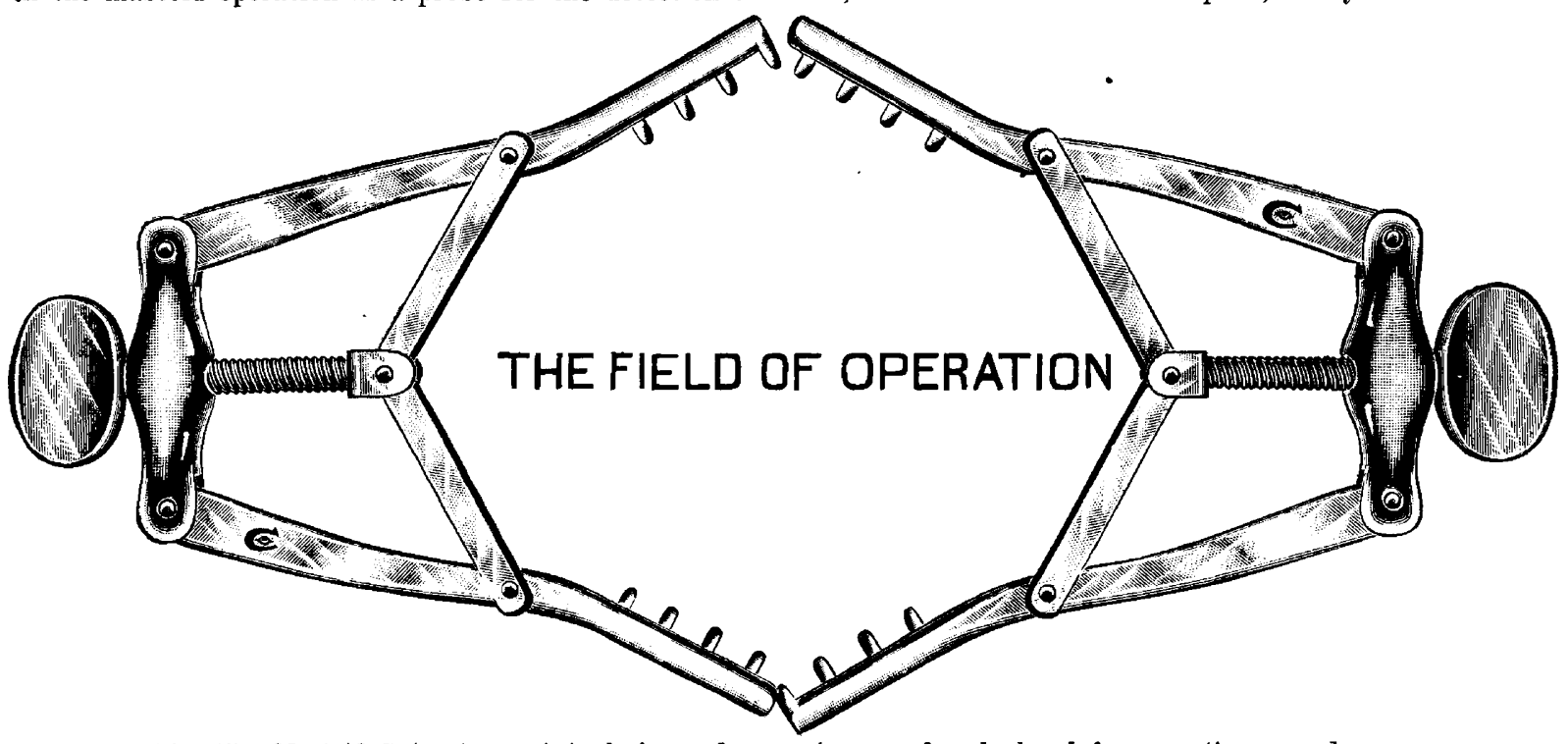

Fig. 15. - Mastoid Retractors. Actual size and properly opened and placed for operative procedures. 
in instrument stores has failed to produce one. I beg, therefore, to suggest one, so simple that apologies seem necessary for the suggestion, and yet one that has seemed to answer satisfactorily the purpose for the past year at St. Luke's Hospital (Fig. 17). It is simply a small tray with a shelf for holding a few bottles, and a space of sufficient size for holding miscellaneous articles such as droppers, gauze, cotton, instruments, etc. I think it undesirable to make many partitions in a small bedside tray like the one under consideration,

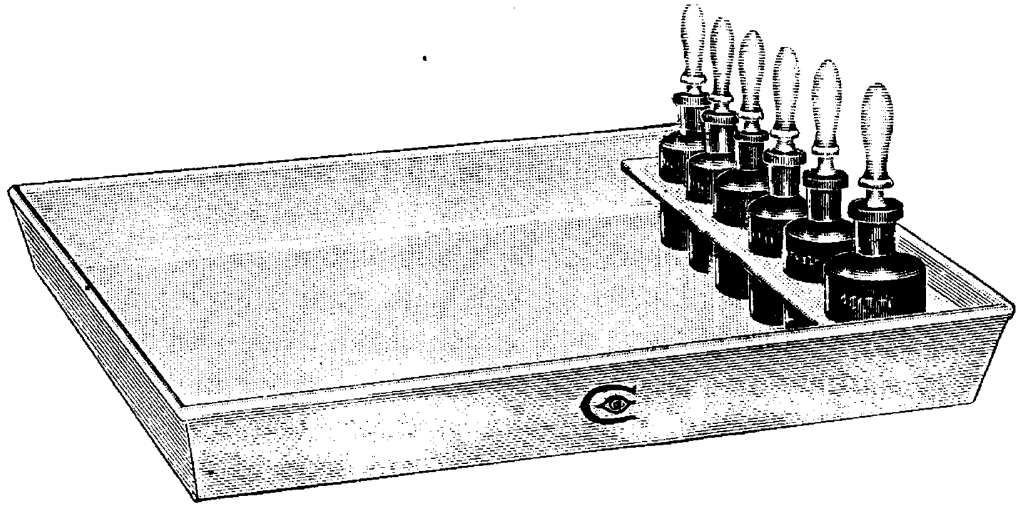

Figure 17.

and have found it much more convenient to lay in the one large space such articles as are absolutely necessary in a tour of the hospital.

\section{A EUSTACHIAN BUR.}

Curetting the tympanic orifice of the Eustachian tube is an important part of the radical mastoid operation, as, if this is not done, granulation tissue usually remains and a mucous secretion from the unclosed tube may become a constant and annoying sequel of the operation. This little procedure may be accomplished with a small, sharp spoon curette, and whiting has devised a conical shaped bur which quickly rims out the orifice. I have, however, found his bur to be over-large for the purpose, and I do not like the conical shape of his bur, as

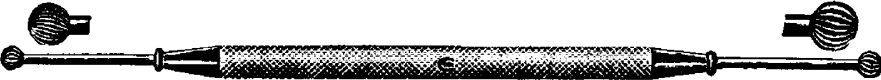

Figure 18.

it may be too energetic in its action, especially when attached to such a strong handle which not only invites undue force, but also obstructs the view of the bur and tube, which should always be kept in sight. I, therefore, use a bur (Fig. 18) with round outlines, which, it would seem, is capable of doing less harm, and also one possessing a straight.handle (without a hand crossbar as in Whiting's), which can be rolled between the fingers when rimming out the tube orifice. It is not likely that much force will be employed in using this instrument, and the bur and tube an a ways be kept clearly in view.

\section{Clinfcal Notes}

\section{TETANUS FOLLOWING VACCINATION \\ A. H. CHURCHILL, M.D. \\ ST. CHARLES, ILL.}

Patient.-E. M. B., aged 51/2 years, female, in perfect health, living in the country on a farm, was vaccinated Jan. 11, 1906, on left arm with P. D. \& Co.'s vaccine from tube. Antiseptic precautions were taken.

History of Disease.-The child was not seen again until January 30, when the following history was obtained: The arm had been but slightly swollen and the child had felt well during the entire time since vaccination. She got up on the morning of January 30, apparently well, sat at table, and ate some breakfast. She seemed to have slight indisposition during the morning, but at noon she drank coffee and milk. During the afternoon the mother said the child wished to lie down and that she noticed some stiffness of the neck.

Examination.-I saw the child at 6:30 p. m. and found some rigidity of the left arm, and considerable rigidity of the neck and jaw. The teeth could be parted about half an inch. There was no rigidity of the right arm or of the back below the neck, or of the legs or abdominal muscles. The child could swallow perfectly.

Course of Disease.-The child was seen again at 1 a. m., January 31. She had had two severe spasms in which she threw herself about in bed, but she was rational when seen. The left arm was some more rigid. The right arm and legs were still-normal. Temperature still normal, and the child could still swallow easily. At this time there were muscular tremors through the abdominal muscles, causing her to cry out with pain. From the onset of the symptoms till death occurred there was no movement of the bowels and no urine was passed. The child rapidly grew weaker antil 9 a. m. on January 31 , when she died, not quite twenty-four hours from the manifestation of the first symptom. There was no postmortem. The arm, on examination, showed vaccination not doing well. A wound half an inch across, edges indurated and undermined, with a foul discharge.

General Remark.-At the time the vaccination was done the arm was scrubbed with soap and water, and then with alcohol. The scarifying was done with a sterile scalpel, and the vaccination was covered with sterile gauze. From that time the parents took care of the arm, as is practically always the case in the country. I did not see the child from the day I did the vaccination till three weeks later. The arm had not been troublesome, so the parents had not thought it necessary to bring the child to my office. Of course, it is possible that the infection gained entrance at some other point, but as the vaccination wound was the only open wound, I considered that must have been the source of infection. I certainly believe that the infection occurred after the vaccination and entirely independent of it, as all authorities, so far as I find, give ten days as the limit of the period of incubation, and this, as I have stated, was just three weeks after I sent to Chicago for antitoxin. The little girl died before this arrived, bowever. I have not seen a case recorded in which death took place so soon after the first manifestations of tetanus.

\section{THE PULMONIC SECOND SOUND, IN HEAL'TH}

AND DISEASE.

\section{IRVIN HARDY, M.D. BALTIMORE}

While due attention has been given to the difference existing between the pulmonic and systemic circulations, as a result of pulmonary consolidation, there seems to be a scarcity of comment regarding a comparative lessening of intra-aortic blood pressure.

By experiment and observation I have found in healthy individuals what appeared to be an accentuated pulmonic second sound which would disappear on having the patient walk briskly for a few minutes.

The point I wish to make is that healthy people who are inactive, engaged in sedentary occupations, have an apparent accentuated pulmonic second when in reality it is only a relative one, due to lessened intra-aortic pressure, the result of deficient exercise. Use is made of exercise to bring out signs of disease of the valve segments, but $I$ have seen no literature bearing on this subject. What theory one may have, the fact remains, or at least is constant enough, to keep one on his guard.

If this be true, it would seem that too much stress has been laid on this sign as an early indication of pulmonary tuberculosis, thus giving an opportunity, at least, for misleading conclusions.

742 West Fayette Street. 